\title{
Electronic states for excess electrons in polyethylene compared to long chain alkanes
}

\author{
David Cubero, Nicholas Quirke \\ Department of Chemistry, Imperial College, London, SW7 2AY, UK \\ David F. Coker \\ Department of Chemistry, Boston University, 590 Commonwealth Avenue, Boston, Mass \\ 02215, USA
}

\begin{abstract}
We use a pseudopotential model to calculate the electronic states available to an excess electron in crystalline and amorphous regions of model polyethlyene (PE) as well as the molecular crystal of the linear alkane $\mathrm{C}_{27} \mathrm{H}_{56}$. It is shown that alkane crystals of whatever chain length are not representative of crystalline polyethylene although they are often considered to be so. We discuss the implications for electron transport in polyethylene.
\end{abstract}


Molecular crystals of linear long-chain alkanes are often used as a model of polyethylene $\left[-\left(\mathrm{CH}_{2}\right)_{\mathrm{x}}-\right]^{1,2,3,4,5}$ due in part to their similar single molecule electronic properties ${ }^{6}$. However in the solid state polyethylene (PE) is not entirely crystalline, but is, to various degrees, semi-crystalline, with coexisting amorphous and crystalline regions ${ }^{1}$. These two regions have different electronic structures, which obscures comparisons between different materials, for example of states available to an excess electron. The most accurate experimental value for the bottom of the conduction band $V_{0}$ in PE comes from photoconduction measurements ${ }^{7}$, which locate $V_{0}$ in the range $0<V_{0}<1 \mathrm{eV}^{8}$. N. Ueno et $a l^{3}$ determined the ground-state energies of excess electron in crystalline long-chain alkanes using low-energy electron transmission and secondary-electron emission experiments. The ground-state energies as a function of the number of carbons in the molecule were found to reach a plateau, practically independent of the chain length (up to n- $\left.\mathrm{C}_{44} \mathrm{H}_{90}\right)$ in the range $0.4-0.6 \mathrm{eV}$. This was taken as an estimate of the bottom of the conduction band in crystalline polyethylene.

Previous theoretical, ab initio and semi-empirical, one-dimensional calculations based on a single chain, give values of the bottom of the conduction band for PE that vary between 0 and $5.8 \mathrm{eV}^{8}$. In a recent density functional theory (DFT) calculation, Righi et $a l^{9}$ reported the values 0.1 and $0.17 \mathrm{eV}$ for the crystalline PE surfaces (001) and (110), respectively. However, their calculation is based on the identification of the Kohn-Sham eigenvalues with excitation energies, which is not a rigorous procedure; it is known that it 
fails to provide the correct band gap in crystalline PE $(8.8 \mathrm{eV})$, with an absolute error larger than $2 \mathrm{eV}^{9}$.

In this letter we present new results for excess electrons in PE and alkane crystals using a fast Fourier transform (FFT) block Lanczos diagonalization algorithm and DFT. A complete description of the methods employed can be found elsewhere ${ }^{10}$. Our semiclassical calculations used classical explicit atom force-field given by Martonak et $\mathrm{al}^{11}$, including bonded (bond stretch, valence angles and dihedrals) and dispersion interactions. The trajectories were generated using the molecular dynamics code DLPOLY ${ }^{12}$. For the electron-molecule interactions we use a semi-empirical pseudopotential, derived by us ${ }^{10}$, to be consistent with experimental data for the density dependence of the threshold $V_{0}$ of conduction in alkane fluids. The pseudopotential consists of a repulsive component constructed from ab initio calculations in methane ${ }^{13}$, which accounts for the interaction with the static charge distribution as well as exchange and orthogonality of the excess electron with the target atom, and a attractive part, which accounts for the polarization interaction between the excess electron and the dielectric. We have used the multi-center polarizabilities reported in Ref. ${ }^{14}$, which were obtained using state of the art ab initio calculations, combined with full many body self-consistent calculations of the polarization interaction as described in Ref. ${ }^{15}$. In all calculations we employed periodic boundary conditions and a cutoff of $9 \AA$. The qualitative differences in excess electronic states we report in this paper arise from differences in molecular geometry in the different materials we study and have nothing to do with small differences in fundamental electronic interactions between eg $\mathrm{CH}_{2}$ and $\mathrm{CH}_{3}$ groups. Note also that corrections due to 
surface effects ${ }^{9}{ }^{10}$ are small, less than $0.1 \mathrm{eV}$, which is also of the same order of the error bars we expect with our pseudopotential.

A fast Fourier transform (FFT) block Lanczos diagonalization algorithm ${ }^{16,17}$ was used to compute the adiabatic excess electronic states of lattice and amorphous configurations. This method provides the lowest energy eigenstates for a given three dimensional potential energy grid, and has been used successfully to describe excess electron in simple fluids ${ }^{15}$. The grid spacing was never bigger than $0.72 \AA$, and we estimate the uncertainty in the results due to grid size as $\sim 0.02 \mathrm{eV}$. In order to improve the sampling at the bottom of the Brillouin zone for periodic systems we have modified the recursion method in order to obtain directly the energy level of a given point $\mathbf{k}$ in the Brillouin zone. According to Bloch's theorem, the Fourier components of the Bloch wave function of wave vector $\mathbf{k}$ will vanish except for the points $\mathbf{k}+\mathbf{G}$, where $\mathbf{G}$ is a vector of the reciprocal space ${ }^{18}$. Therefore, the corresponding Bloch function can be directly calculated if all the other Fourier components are set to zero at every step of the recursion algorithm. In practice, we kept the components corresponding to the desired $\mathbf{k}$ and all the symmetry equivalents ${ }^{19}$, which provide a set of degenerate eigenstates.

The crystalline regions of PE have been modeled as a perfect orthorhombic lattice with unit cell parameters taken from ray diffraction data at room temperature 20212223 . The caxis was chosen to be in the direction of the chains (see Fig. 1). The modified Fourier transform (FFT) block Lanczos diagonalization algorithm applied to the PE lattice using our new pseudopotential predicts a ground state energy for the excess electron of $E_{0}=0.65 \mathrm{eV}$ and a kinetic energy of $2.4 \mathrm{eV}$. Since we have a perfect periodic system, 
this state can be identified as the bottom of the conduction band. In Fig. 1 (a) we present a volumetric representation of the excess electron probability density for this state. The wave function is clearly interchain, in agreement with the results presented by S. Serra et $a l^{24}$. Fig. 1 (b) shows the conduction band dispersion of crystalline PE along several lines in the first Brillouin zone ${ }^{19}$. As a reference, we have also plotted the energy levels of a free excess electron $\varepsilon_{\mathbf{k}-\mathbf{G}}^{0}=\mathrm{h}^{2}(\mathbf{k}-\mathbf{G})^{2} / 2 m_{e}$. The results show that the band dispersion is strongly free electron like, with an effective mass of $m^{*}=1.0 m_{e}$ along the $c$-axis, where $m_{e}$ is the mass of the electron. The effective mass tensor shows anisotropy, with effective masses of $1.7 m_{e}$ and $1.4 m_{e}$ along the $a$ and $b$ axis, respectively.

The long-chain alkane n- $\mathrm{C}_{27} \mathrm{H}_{56}$ has a stable orthorhombic structure at room temperature similar to crystalline PE, but with the molecules packed in layers due to their finite length. Adjacent layers are shifted ${ }^{25}$ along the a-axis by $2.5 \AA$. Applying our methods to a molecular crystal of $n-\mathrm{C}_{27} \mathrm{H}_{56}$ we find a ground state energy for the excess electron of $E_{0}=0.39 \mathrm{eV}$. This value compares favorably with the experimental data ${ }^{3}$ which are in the range $0.4-0.6 \mathrm{eV}$ but is $0.26 \mathrm{eV}$ below the lowest conducting state of crystalline PE. The reason for this difference is clear from the plot of the excess electron density in Figure 2. The bottom of the conduction band is not interchain, as in PE, but interlayer. The electron is localized in the gap separating the layers. The main contribution to the energy difference comes from the kinetic energy $\left(K=2.0 \mathrm{eV}\right.$ for n- $\left.\mathrm{C}_{27} \mathrm{H}_{56}\right)$. In the molecular crystal the excess electron finds more free volume in the interlayer gap, and as a consequence a lower kinetic energy. Moreover, the ground state is nearly degenerate, 
each state corresponding to localization in each available interlayer gap, which is in contrast with the free electron-like band found in crystalline PE. The gap separation between the planes of the terminal $\mathrm{CH}_{3}$ groups of alkanes with an odd number of carbons is $3.148 \AA^{26}$. Even-numbered long-chain alkanes are found to crystalize all-trans in simple layers with a monoclinic or triclinic unit cells, but with the same gap separation of about $3 \AA^{26}$. Since the ground state depends mainly on this separation, the ground state energies of different long-chain alkanes should all be very close to each other, though different to crystalline PE. Moreover, in the later electron transport is expected to be strongly anisotropic (with a preferred direction parallel to the chains), whereas in the alkane crystal it is at an angle to the chain direction (along the interlayer gap) with additional conduction parallel to the chains occurring at the excited state $\sim 0.26 \mathrm{eV}$ above the bottom of the conduction band.

We can use the calculated value of the effective mass to compute the order of magnitude of the electron mobility given by band conduction in crystalline PE. Using the expression $\mu=e \tau / m^{*}$ and taking the electron collision time as $\tau=\lambda / \nu$, with the electronic velocity $v$ estimated from the (room) temperature, and setting $\lambda=2.53 \AA$ (minimum length parameter), we obtain a lower bound of $\mu \geq 1 \mathrm{~cm}^{2} / \mathrm{V} \cdot \mathrm{s}$. This value is too high in comparison with the reported mobility in real (that is partially disordered) PE, which is in the range $10^{-3}-10^{-10} \mathrm{~cm}^{2} / \mathrm{V} \cdot \mathrm{s}{ }^{27} 28$. However, both in simple crystals of long-chain alkanes ${ }^{29}$ and in photoconduction experiments in $\mathrm{PE}^{7}$ (where electrons are excited into the conduction band in the crystalline regions) a much larger mobility is observed. The 
much smaller mobility in PE in absence of irradiation, is probably a sign that conduction in the amorphous regions of PE plays a very important role.

Anaylisis of the excess electronic states in amorphous PE shows a band tail of localized states, with the bottom of the band located below the vacuum level, at about $-0.3 \mathrm{eV}^{10}$. This is in agreement with experiments on molten long-chain alkanes, where the ground state energy was found to be negative $\mathrm{e}^{3}$. A careful analysis of the eigenstates shows a transition from localized to extended states at about the vacuum level ${ }^{10}$, implying an activation energy of $0.3 \mathrm{eV}$, which is in good agreement with the trap depth of $0.35 \mathrm{eV}$ attributed to amorphous regions of PE estimated from thermoluminescence and thermally stimulated conductivity measurements ${ }^{30} 31$, as well as the activation energy of $0.24 \mathrm{eV}$ obtained from mobility measurements ${ }^{32}$.This is well below the bottom of the band for crystalline PE and therefore the localized states discussed above cannot be considered a perturbation or a band tail of crystalline states which are not present.

In conclusion, we have shown that even though crystalline PE and alkane crystals present a similar structure and negative electron-affinity, conduction in the two materials is found to be quite different; in PE conduction is interchain, in an alkane crystal it is interlayer. Nevertheless, long-chain alkanes in a disordered state are still expected to be a good model of amorphous regions of polyethylene, where the electronic states are governed by the density fluctuations of the material. In subsequent papers we will explore the electron trajectories on these energy surfaces using surface hopping techniques. 
From these preliminary results we can speculate that in polyethylene with a high degree of crystallinity, amorphous and interfacial regions constitute electron traps of the order of $1 \mathrm{eV}$ (for example adding the value of the bottom of the conduction band in the crystal and the ground state of the amorphous region we have $+0.65+0.3=0.95 \mathrm{eV}$ ) and are therefore likely to play an important role in determining electron transport in polyethylene (along with the chemical traps ${ }^{33},{ }^{34}$ ). This is in agreement with the experimental results by Tanaka ${ }^{35}$, who found an optical gap of $7.35 \mathrm{eV}$ with a band tail value around $1 \mathrm{eV}$. On the other hand, in low density polyethylene the energy difference of $0.65 \mathrm{eV}$ between the conduction levels in the crystalline and amorphous regions would prevent the electron from penetrating the crystalline regions and conduction would only occur through the extended states at the amorphous regions. The details of these effects and the role of structural inhomogeneities in determining the current voltage characteristics of PE will be the subject of future work.

\section{Acknowledgements}

This work was supported by EPSRC through grants GR/R18222 and GR/M94427. DFC acknowledges support through a Schlumberger visiting Professorship of Chemistry at the University of Cambridge. 



\section{List of figures}

Figure 1: (a) Orthographic view of the ground state wave function for crystalline PE. The excess electron is depicted using a volumetric representation of the density probability. The lighter regions correspond to the higher density values, showing the interchain nature of the conducting state. (b) Conduction band dispersion along symmetry directions in crystalline PE (diamonds). Lines correspond to a free electron.

Figure 2: Ground-state wavefunction for crystalline $n-\mathrm{C}_{27} \mathrm{H}_{56}$. Orthographic views along the c-axis (a) and the b-axis (b) showing the interlayer gap. The wave function is clearly interlayer, rather than interchain. 


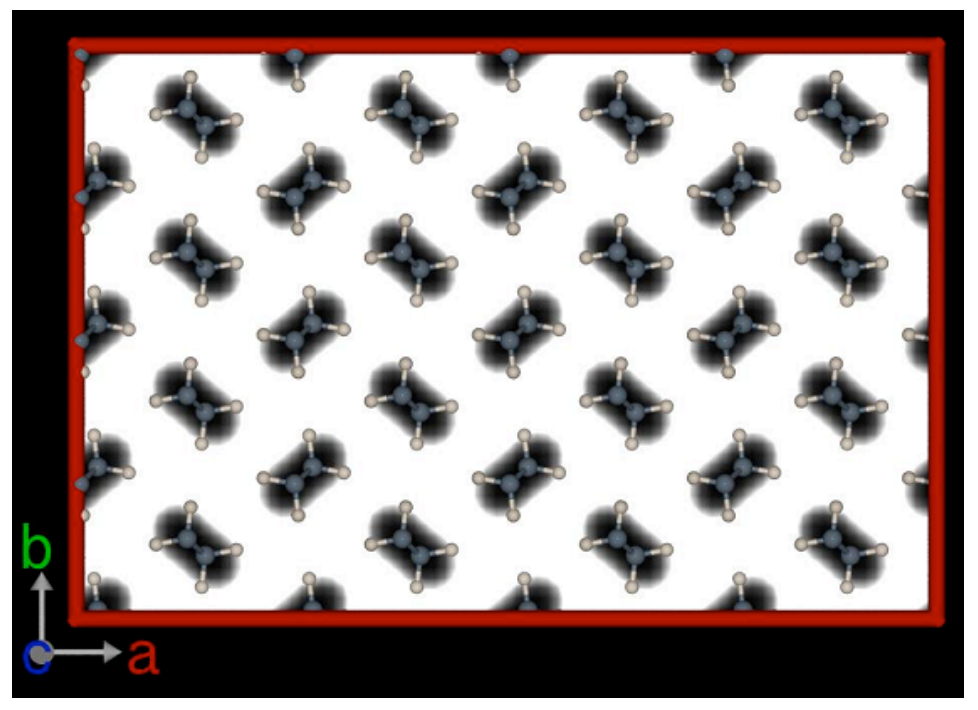

(a)

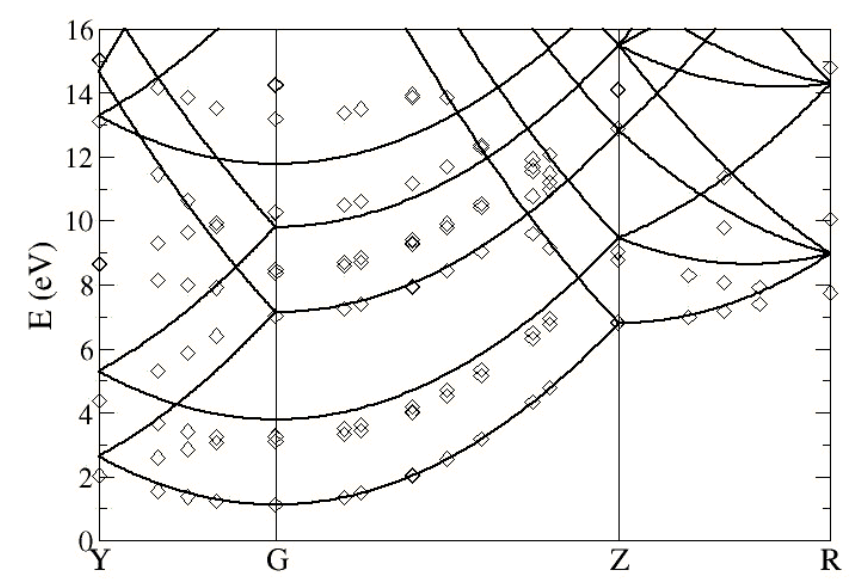

(b)

Figure 1: (a) Orthographic view of the ground state wave function for crystalline PE. The excess electron is depicted using a volumetric representation of the density probability. The lighter regions correspond to the higher density values, showing the interchain nature of the conducting state. (b) Conduction band dispersion along symmetry directions in crystalline PE (diamonds). Lines correspond to a free electron. 


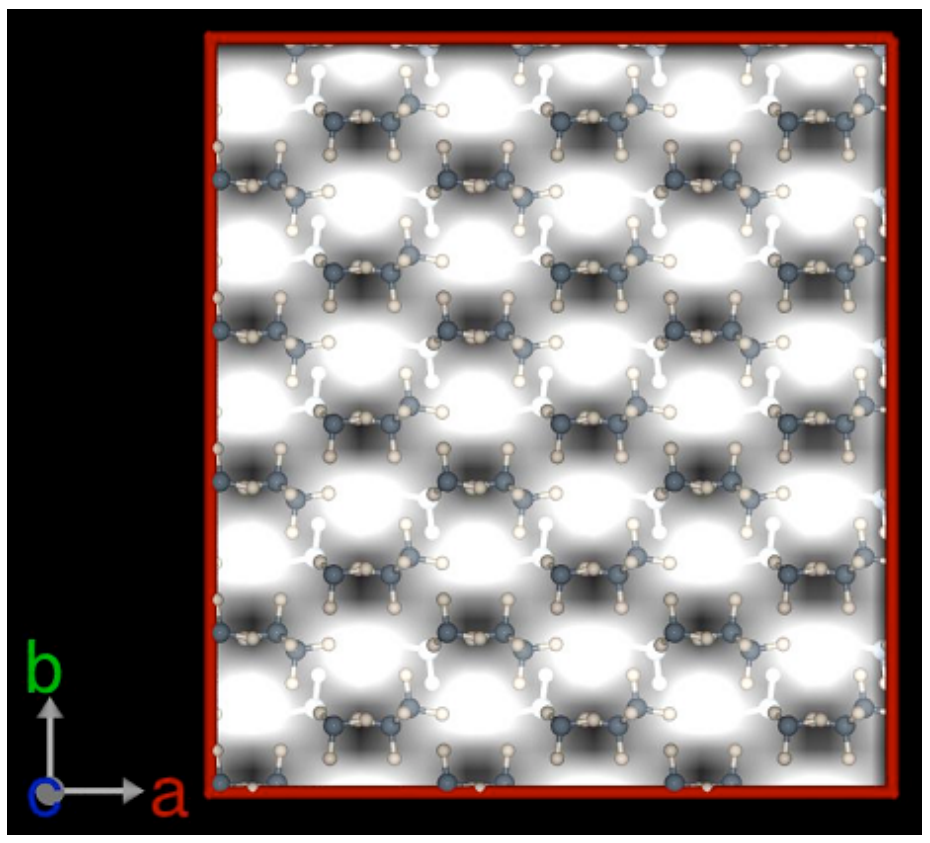

(a)

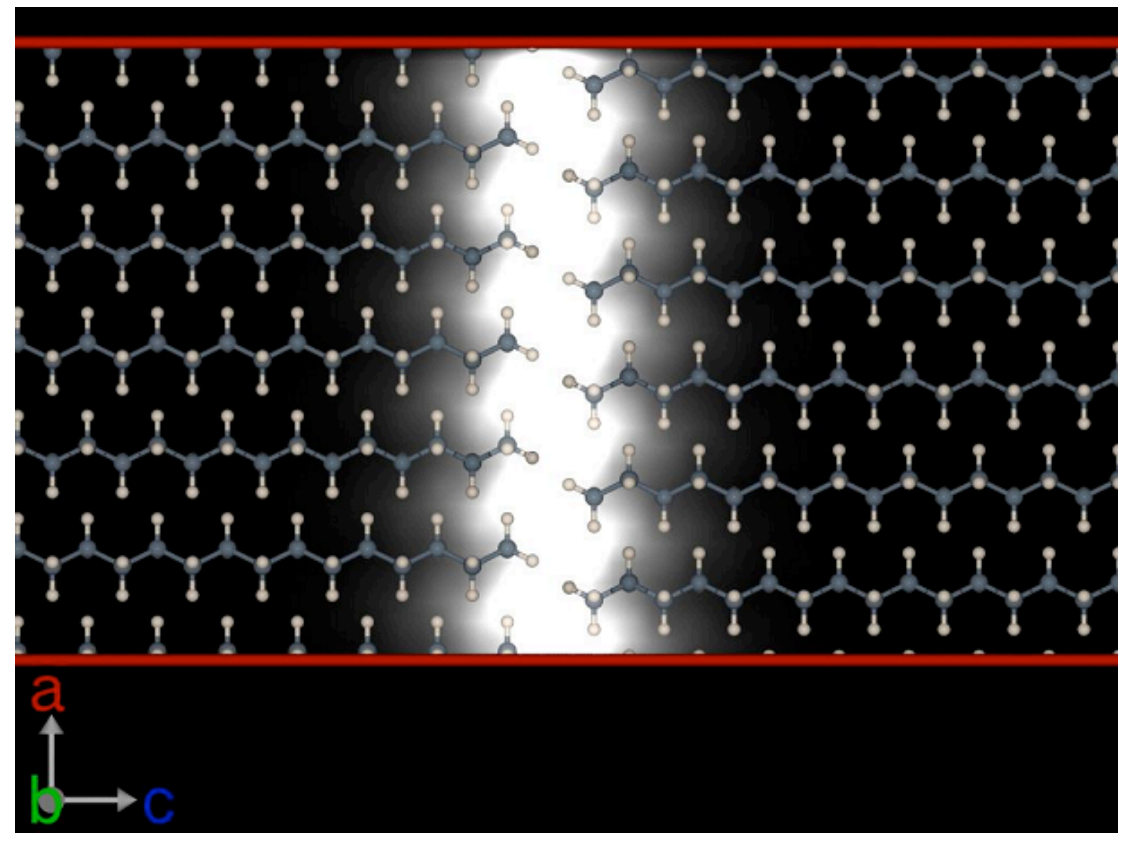

(b)

Figure 2: Ground-state wavefunction for crystalline $n-\mathrm{C}_{27} \mathrm{H}_{56}$. Orthographic views along the c-axis (a) and the b-axis (b) showing the interlayer gap. The wave function is clearly interlayer, rather than interchain. 
${ }^{1}$ L. A. Dissado and J. C. Fothergill, Electrical degradation and breakdown in polymers, Peter Peregrinus, Stevenage, 1992.

${ }^{2}$ D. M. Taylor and I. E. Noble, J. Phys. D: Appl. Phys. 13 (1980) 2123.

${ }^{3}$ N. Ueno, K. Sugita, K. Seki, and H. Inokuchi, Phys. Rev. B 34 (1986) 6386.

${ }^{4}$ E. Cartier and P. Pfluger, IEEE Trans. Electr. Ins. EI-22 (1987) 123.

${ }^{5}$ R. Dudde and B. Reihl, Chem. Phys. Lett. 196 (1992) 91.

${ }^{6}$ J. J. Pireaux, Phys. Rev. A 14 (1976) 2133.

${ }^{7}$ K. J. Less and E. G. Wilson, J. Phys. C.: Solid State Phys. 6 (1973) 3110.

${ }^{8}$ D. Bloor, Chem. Phys. Lett. 40 (1976) 323.

${ }^{9}$ M. C. Righi, S. Scandolo, S. Serra, S. Iarlori, E. Tosatti, and G. Santoro, Phys. Rev. Lett. 87 (2001) 076802-1.

${ }^{10}$ D. Cubero, D. F. Coker and N. Quirke (to be published).

${ }^{11}$ R. Martonak, W. Paul, and K. Binder, J. Chem. Phys. 106 (1997) 8918.

${ }^{12}$ W. Smith and T. Forester, J. Molec. Graphics 14 (1996) 136.

${ }^{13}$ Z. Liu and B. J. Berne, J. Chem. Phys. 99 (1993) 9054.

${ }^{14}$ S. Nakagawa, Chem. Phys. Lett. 278 (1997) 272.

${ }^{15}$ B. Space, D. F. Coker, Z. H. Lui, B. J. Berne, and G. Martyna, J. Chem. Phys. 97 (1992) 2002.

${ }^{16}$ F. Webster, P. J. Rossky, and R. A. Friesner, Comp. Phys. Comm. 63 (1991) 492.

${ }^{17}$ M. H. Gutknecht, Acta Numerica, Cambridge University Press, Cambridge, 1997.

${ }^{18}$ N. W. Ashcroft and N. D. Mermin, Solid State Physics, Saunders, Fort Worth, 1976. 
${ }^{19}$ C. J. Bradley and A. P. Cracknell, The mathematical theory of symmetry in solids, Clarendon, Oxford, 1972.

${ }^{20}$ C.W.Bunn, Trans. Faraday Soc. 35 (1939) 482.

21 S. Kavesh and J. M. Schultz, J. Polym. Sci. Part A-2 8 (1970) 243.

${ }^{22}$ H. Shearer and V. Vand, Acta Cryst. 9 (1956) 3379.

${ }^{23}$ R. Martonak, W. Paul, and K. Binder, J. Chem. Phys. 106 (1997) 8918.

${ }^{24}$ S. Serra, E. Tosatti, and S. Iarlori, Phys. Rev. B 62 (2000) 4389.

${ }^{25}$ A. E. Smith, J. Chem. Phys. 21 (1953) 2229.

${ }^{26}$ M. Dirand, M. Bouroukba, V. Chevallier, and D. Petitjean, J. Chem. Eng. Data 47 (2002)115.

${ }^{27}$ M. Ieda, IEEE Trans. Electr. Ins. EI-19 (1984) 162.

${ }^{28}$ It has been suggested that the striking range of variation of the mobility values reported by various investigators is mainly due to the different measurement procedures adopted, and in particular to the different measurement times, see T. Tanaka, IEEE Trans. DEI 8 (2002) 733.

${ }^{29}$ K. Yoshino, S. Harada, J. Kyokane, and Y. Inuishi, J. Phys. D: Appl. Phys. 12 (1979) 1535.

${ }^{30}$ T. Nishitani, K. Yoshino, and Y. Inuishi, Jpn. J. Appl. Phys. 14 (1975) 721.

${ }^{31}$ T. Nishitani, K. Yoshino, and Y. Inuishi, Trans. Inst. Electr. Eng. Jpn. 96-A (1976) 381.

${ }^{32}$ T. Tanaka and J. H. Calderwood, J. Phys. D: Appl. Phys. 7 (1974) 1294.

${ }^{33}$ M. Meunier, A. Aslanides and N. Quirke, J Chem Phys 115 (2001) 2876.

${ }^{34}$ J. A. Anta, G. Marcelli, M. Meunier, and N. Quirke, J. Appl. Phys. D 92 (2002) 1002. 
${ }^{35}$ T. Tanaka, J. Appl. Phys. 44 (1973) 2430. 